NASA/CR-2002-211657

ICASE Report No. 2002-17

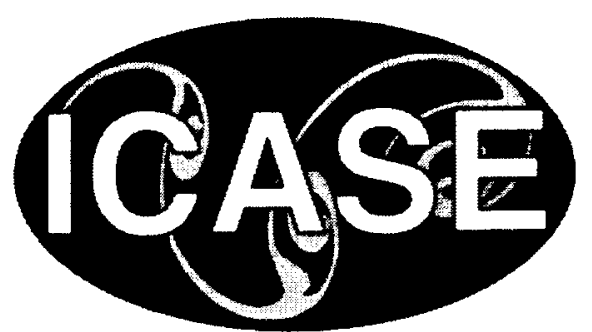

\title{
Lattice Boltzmann Method for 3-D Flows with Curved Boundary
}

Renwei Mei, Wei Shyy, and Dazhi Yu

University of Florida, Gainesville, Florida

Li-Shi Luo

ICASE, Hampton, Virginia

ICASE

NASA Langley Research Center

Hampton, Virginia

Operated by Universities Space Research Association 
Available from the following:

NASA Center for AeroSpace Information (CASI) 7121 Standard Drive

Hanover, MD 21076-1320

(301) 621-0390
National Technical Information Service (NTIS) 5285 Port Royal Road

Springfield, VA 22161-2171

(703) 487-4650 


\title{
LATTICE BOLTZMANN METHOD FOR 3-D FLOWS WITH CURVED BOUNDARY
}

\author{
RENWEI MEI*, WEI SHYY ${ }^{\dagger}$, DAZHI YU ${ }^{\ddagger}$, AND LI-SHI LL: $O^{\S}$
}

\begin{abstract}
In this work, we investigate two issues that are important to computational efficiency and reliability in fluid dynamics applications of the lattice Boltzmann equation (LBE): (1) Computational stability and accuracy of different lattice Boltzmann models and (2) the treatment of the boundary conditions on curved solid boundaries and their 3-D implementations. Three athermal 3-D LBE models (D3Q15, D3Q19, and D3Q27) are studied and compared in terms of efficiency, accuracy, and robustness. The boundary treatment recently developed by Filippova and Hänel and Mei et al. in 2-D is extended to and implemented for 3-D. The convergence, stability, and computational efficiency of the 3-D LBE models with the boundary treatment for curved boundaries were tested in simulations of four 3-D flows: (1) Fully developed flows in a square duct, (2) flow in a 3-D lid-driven cavity, (3) fully developed flows in a circular pipe, and (4) a uniform flow over a sphere. We found that while the fifteen-velocity 3-D (D3Q15) model is more prone to numerical instability and the D3Q27 is more computationally intensive, the D3Q19 model provides a balance between computational reliability and efficiency. Through numerical simulations, we demonstrated that the boundary treatment for 3-D arbitrary curved geometry has second-order accuracy and possesses satisfactory stability characteristics.
\end{abstract}

Key words. lattice Boltzmann method, boundary condition for curved geometries, accuracy, 3-D flows, Navier-Stokes equations

Subject classification. Fluid Dynamics

\section{Introduction.}

1.1. Basic Notion of the Lattice Boltzmann Equation. In one fashion or another, conventional methods of computational fluid dynamics (CFD) compute pertinent flow fields, such as velocity $u$ and pressure $p$, by numerically solving the Navier-Stokes equations in space $x$ and time $t[23,9,27]$. In contrast, various kinetic methods use the transport equation, or the Boltzmann equation in particular, for various problems in fluid dynamics. The Boltzmann equation deals with the single particle distribution function $f(\boldsymbol{x}, \boldsymbol{\xi}, t)$, where $\boldsymbol{\xi}$ is the particle velocity, in phase space $(\boldsymbol{x}, \boldsymbol{\xi})$ and time $\boldsymbol{t}$. Recently, the method of the lattice Boltzmann equation (LBE) $[5,24,3,6]$ has become an alternative to the conventional CFD methods employing Navier-Stokes equations. The theoretical premises of the LBE method are that (1) hydrodynamics is insensitive to the details of microscopic physics, and (2) hydrodynamics can be preserved so long as the conservation laws and associated symmetries are respected in the microscopic or mesoscopic level. Therefore, the computational advantages of the LBE method are attained by drastically reducing the particle velocity

\footnotetext{
*Department of Aerospace Engineering, Mechanics \& Engineering Science, University of Florida, Gainesville, FL $32611-6250$ (email address: rwmQaero.ufledu).

${ }^{\dagger}$ Department of Aerospace Engineering, Mechanics \& Engineering Science, University of Florida, Gainesville, FL 32611-6250 (emajl address: wsQaero.ufl.edu).

$\ddagger$ Department of Aerospace Engineering, Mechanics \& Engineering Science, University of Florida, Gainesville, FL 32611-6250 (email address: ydz@aero.ufl.edu).

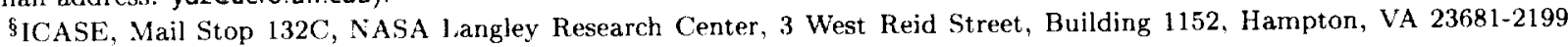
(email address: luo@icase edu). This research was supported by the National Aeronautics and Space Administration under NASA Contract No. NAS1-97046 while the author was in residence at ICASE, NASA Langley Research Center, Hampton, VA 23681-2199.
} 
space $\boldsymbol{\xi}$ to only a very fow discrete points without seriously degrading hydrodynamics. This is possible because the LBE method rigorously preserves the hydrodynamic moments of the distribution function $f$, such as mass density and momentum fluxes, and the necessary symmetries $[13,14,1]$.

One popular kinetic model is the Boltzmann equation with the single relaxation time approximation [4]:

$$
\partial_{t} f+\boldsymbol{\xi} \cdot \nabla f=-\frac{1}{\lambda}\left[f-f^{(0)}\right]
$$

where $\boldsymbol{\xi}$ is the particle velocity, $f^{(0)}$ is the equilibrium distribution function (the Maxwell-Boltzmann distribution function), and $\lambda$ is the relaxation time. The mass density $\rho$ and momentum density $\rho \boldsymbol{u}$ are the first $(D+1)$ hydrodynamic moments of the distribution function $f$ and $f^{(0)}$, where $D$ is the dimension of velocity space.

To solve for $f$ numerically, Eq. (1.1) is first discretized in the velocity space $\boldsymbol{\xi}$ using a finite set of velocities $\left\{\boldsymbol{\xi}_{\alpha}\right\}$ without affecting the conserved hydrodynamic moments $[13,14,1]$,

$$
\partial_{t} f_{\alpha}+\boldsymbol{\xi}_{\alpha} \cdot \nabla f_{\alpha}=-\frac{1}{\lambda}\left[f_{\alpha}-f_{\alpha}^{(0)}\right] .
$$

In the above equation, $f_{\alpha}(\boldsymbol{x}, t)=f\left(\boldsymbol{x}, \boldsymbol{\xi}_{\alpha}, t\right)$ and $f_{\alpha}^{(0)}(\boldsymbol{x}, t)=f^{(0)}\left(\boldsymbol{x}, \boldsymbol{\xi}_{\alpha}, t\right)$ are the distribution function and the equilibrium distribution function of the $\alpha$-th discrete velocity $\xi_{\alpha}$, respectively. The nine-velocity (or 9-bit) LBE model on the 2-D square lattice, denoted as D2Q9 model, has been widely used for simulating 2-D flows. For 3-D flows, there are several cubic lattice models, such as the fifteen-velocity (D3Q15), nineteenvelocity (D3Q19), and twenty-seven-velocity (D3Q27) models [12], which have been used in the literature. All three models have a rest particle (with zero velocity) in the discretized velocity set $\left\{\boldsymbol{\xi}_{\alpha}\right\}$. A minor variation of those models is to remove the rest particles from the discrete velocity set; the resulting models are known as the D3Q14, D3Q18, and D3Q26 models, respectively. The LBE models with a rest particle generally have better computational stability. For athermal fluids, the equilibrium distributions for D2Q9, D3Q15, D3Q19, and D3Q27 models are all of the form [14]

$$
f_{\alpha}^{(e q)}=w_{\alpha} \rho\left[1+\frac{3}{c^{2}} e_{\alpha} \cdot u+\frac{9}{2 c^{4}}\left(e_{\alpha} \cdot u\right)^{2}-\frac{3}{2 c^{2}}(u \cdot u)\right]
$$

where $w_{\alpha}$ is a weighting factor and $e_{\alpha}$ is a discrete velocity, $c=\delta x / \delta t$ is the lattice speed, and $\delta x$ and $\delta t$ are the lattice constant and the time step, respectively. (The values of the weighting factor $w_{\alpha}$ for D3Q15, D3Q19, and D3Q27 models and the diagrams illustrating the lattice structure for D3Q15 and D3Q19 models are given in the Appendix.) It can be shown that $f_{\alpha}^{(e q)}$ is in fact a Taylor series expansion of the Maxwellian $f_{\alpha}^{(0)}[13,14]$. This approximation of $f_{\alpha}^{(0)}$ by the above $f_{\alpha}^{(e q)}$ makes the method valid only in the incompressible limit $u / c \rightarrow 0$.

With the velocity space discretized, the hydrodynamic moments of $f$ and $f^{(0)}$ are evaluated by the following quadrature formulas:

$$
\begin{aligned}
& \rho=\sum_{\alpha} f_{\alpha}=\sum_{\alpha} f_{\alpha}^{(0)}, \\
& \rho \boldsymbol{u}=\sum_{\alpha} \boldsymbol{e}_{\alpha} f_{\alpha}=\sum_{\alpha} \boldsymbol{e}_{\alpha} f_{\alpha}^{(0)} .
\end{aligned}
$$

The speed of sound of the above 3-D LBE models is $c_{s}=c / \sqrt{3}$ and the equation of state is that of an ideal gas $p=\rho c_{s}^{2}$. The viscosity of the fluid is $\nu=\lambda c_{s}^{2}$.

Equation (1.2) is often discretized in space $\boldsymbol{x}$ and time $t$ into

$$
f_{\alpha}\left(\boldsymbol{x}_{i}+\boldsymbol{e}_{\alpha} \delta t, t+\delta t\right)+f_{\alpha}\left(\boldsymbol{x}_{i}, t\right)=-\frac{1}{\tau}\left[f_{\alpha}\left(\boldsymbol{x}_{i}, t\right)-f_{\alpha}^{(0)}\left(\boldsymbol{x}_{i}, t\right)\right]
$$


where $\tau=\lambda / \delta t$. This is the lattice Boltzmann equation with the Bhatnagar-Gross-Krook (BGK) approximation [4] and is often referred to as the LBGK model $[5,24]$. The viscosity in the NS equation derived from Eq. (1.5) is

$$
\nu=(\tau-1 / 2) c_{s}^{2} \delta t
$$

This choice for the viscosity makes the LBGK scheme formally a second order method for solving incompressible flows [14]. The positivity of the viscosity requires that $\tau>1 / 2$. Equation (1.5) can be solved in the following two steps:

$$
\begin{array}{ll}
\text { collision step: } & \tilde{f}_{\alpha}\left(\boldsymbol{x}_{i}, t\right)=f_{\alpha}\left(\boldsymbol{x}_{i}, t\right)-\frac{1}{\tau}\left[f_{\alpha}\left(\boldsymbol{x}_{i}, t\right)-f_{\alpha}^{(0)}\left(\boldsymbol{x}_{i}, t\right)\right] . \\
\text { streaming step: } & f_{\alpha}\left(\boldsymbol{x}_{\boldsymbol{i}}+\boldsymbol{e}_{\alpha} \delta t, t+\delta t\right)=\tilde{f}_{\alpha}\left(\boldsymbol{x}_{i}, t\right),
\end{array}
$$

where $\tilde{f}_{\alpha}$ denotes the post-collision state of the distribution function. It is noted that the collision step is completely local, and the streaming step is uniform and requires little computational effort. Equation (1.7) is explicit, easy to implement, and straightforward to parallelize.

1.2. Boundary Condition on a Solid Surface. To date, most Neumann type boundary conditions for a solid boundary used in the LBE method are based upon the bounce-back boundary condition: A particle colliding with a stationary wall simply reverses its momentum. Much of the previous work on LBE boundary conditions is devoted to the analysis and improvement of the bounce-back boundary condition $[29,10,17,2,22,7,11,15,19,30]$. The bounce-back boundary condition can attain second-order accuracy if the boundary is fictitiously placed half-way between two nodes. That is, the second-order accuracy of the bounce-back boundary condition can only be achieved when the boundaries are located right in the middle of two neighboring lattices $[\Delta=0.5$; see Eq. (1.8)]. (Readers are referred to our recent work [20] for a summary of the previous work.) This prevents the direct application of the bounce-back type boundary conditions to simulate a solid body with smooth curvature. To circumvent this difficulty, Mei \& Shyy solved Eq. (1.2) in curvilinear coordinates using a finite difference method to solve for $\tilde{f}_{\alpha}[21]$. One can also use body-fitted curvilinear coordinates with interpolation throughout the entire mesh, except at the boundaries where the bounce-back boundary condition is used [12]. In more recent works [8, 20], Cartesian coordinates are adopted with interpolation used only at the boundaries. These techniques rely on the freedom of using interpolation techniques. We used the latter technique in the present work.

As shown in Fig. 1 for a 2-D projection involving a 3-D body, the streaming step requires the knowledge of $\tilde{f}_{\bar{\alpha}}\left(\boldsymbol{x}_{b}, t\right)$, in which $\boldsymbol{e}_{\bar{\alpha}}=-\boldsymbol{e}_{\alpha}$, at $\boldsymbol{x}_{b}$ on the solid side in order to compute $\tilde{f}_{\bar{\alpha}}\left(\boldsymbol{x}_{f}, t\right)$ for the lattice node located on the fluid side at $x_{f}=x_{b}+e_{\bar{\alpha}} \delta t$. Defining

$$
\Delta=\frac{\left\|x_{f}-x_{w}\right\|}{\left\|x_{f}-x_{b}\right\|}
$$

as the fraction of an intersected link in the fluid region, it is seen that $0 \leq \Delta \leq 1$ and the horizontal or vertical distance between $x_{b}$ and $x_{w}$ is $(1-\Delta) \delta x$ on the cubic lattice.

Based on the work of Filippova and Hänel [8], hereinafter referred to as FH, Mei et al. [20] proposed the following treatment for on curved boundaries,

$$
\tilde{f}_{\bar{\alpha}}\left(\boldsymbol{x}_{b}, t\right)=(1-\chi) \tilde{f}_{\bar{\alpha}}\left(\boldsymbol{x}_{f}, t\right)+\chi f_{\kappa}^{(*)}\left(\boldsymbol{x}_{b}, t\right)+2 w_{\alpha} \rho \frac{3}{2 c^{2}} \boldsymbol{e}_{\bar{\alpha}} \cdot \boldsymbol{u}_{w}
$$

with

$$
f_{\alpha}^{(*)}\left(\boldsymbol{x}_{b}, t\right)=w_{\alpha} \rho\left[1+\frac{3}{c^{2}} \boldsymbol{e}_{\alpha} \cdot \boldsymbol{u}_{b f}+\frac{9}{2 c^{4}}\left(\boldsymbol{e}_{\alpha} \cdot \boldsymbol{u}_{f}\right)^{2}-\frac{3}{2 c^{2}}\left(\boldsymbol{u}_{f} \cdot \boldsymbol{u}_{f}\right)\right]
$$




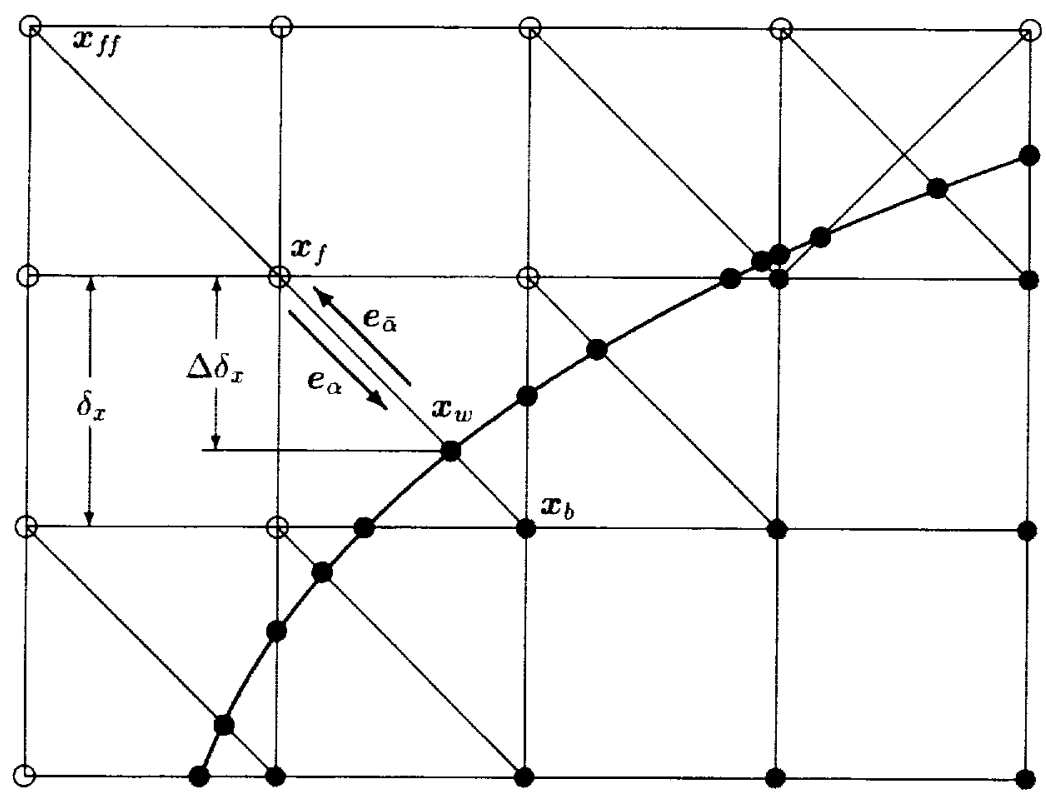

FIG. 1. 2-D projection of the layout of the regularly spaced lattices and curved wall boundary. The thick curve marks the boundary location. The solid circles $(\bullet)$ mark the positions where particle-boundary collision occurs. The empty ( $(0)$ and shaded (•) circles are fluid sites and solid sides, respectively.

and

$$
\begin{array}{lll}
\boldsymbol{u}_{b f}=\frac{(\Delta-1)}{\Delta} \boldsymbol{u}_{f}+\frac{1}{\Delta} \boldsymbol{u}_{w} & \text { and } \quad \chi=\frac{1}{\tau}(2 \Delta-1) & \text { for } \Delta \geq 1 / 2, \\
\boldsymbol{u}_{b f}=\boldsymbol{u}_{f f}=\boldsymbol{u}_{f}\left(\boldsymbol{x}_{f}+\boldsymbol{e}_{\bar{\alpha}} \delta t, t\right) & \text { and } \quad \chi=\frac{(2 \Delta-1)}{(\tau-2)} & \text { for } \Delta<1 / 2 .
\end{array}
$$

It is noted that Eq. (1.11b) for $\boldsymbol{u}_{b f}$ and $\chi$ differs from that originally proposed by FH. The choice for $\boldsymbol{u}_{b f}$ given by Eq. (1.11b) improves the computational stability for $\tau<1$ and $\Delta<1 / 2$ [20]. Since Eqs. (1.9) (1.11) are in vector form, they can be directly extended to 3-D flows with curved boundaries.

1.3. Scope of the Present Work. The present study examines two issues in 3-D incompressible fluid dynamics simulations with arbitrary boundaries using the LBE method: i) The performance of various 3-D athermal LBE models for viscous flows, and ii) the efficacy and reliability of the extension of the curved boundary treatment from 2-D to 3-D flows. We focus on the stability and accuracy of the computation and the robustness in handling an arbitrary curved geometry. In Section 2, a modification of the choice of $\boldsymbol{u}_{b f}$ and the expression for $\chi$ when $\Delta \geq 1 / 2$ is proposed in order to further improve the computational stability of the boundary treatment. In Section 3, numerical results for four 3-D steady flows are examined and various computational issues are addressed. These four cases are: (i) pressure driven fully developed flow in a square duct; (ii) 3-D lid-driven cavity flow; (iii) pressure driven fully developed flow in a circular pipe; and (iv) uniform flow over a sphere. In cases (i) and (iii), the LBE-based numerical solutions can be compared with known exact solutions so that the accuracy of the LBE solutions can be determined. The difference in these two cases is that $\Delta$ is a constant in the square duct while $\Delta$ varies around the solid boundary in the circular pipe. In the lid-driven cavity flow, the singularity at the corners between the moving and stationary walls allows for a performance assessment of various LBE schemes. The flow past a sphere is an external flow around a 3-D blunt body. In all four cases, detailed assessments are made in terms of error norms and velocity profiles. It will be demonstrated that accurate and robust solutions are obtained using the newly 
proposed boundary conditions along with the selected LBE models.

2. Modification of the Boundary Condition for $\Delta>1 / 2$. Equations (1.9) (1.11) are first applied to a fully developed pressure driven 2-D channel flow by using the 3-D LBE model D3Q19. At the inlet ( $i=1)$ and exit ( $i=N_{x}$, in which $N_{x}$ is the number of lattices in the $x$-direction) the following zero derivative condition is imposed after the collision step,

$$
\begin{aligned}
& \tilde{f}_{\alpha}(i=1, j, k)=\tilde{f}_{\alpha}(i=2, j, k), \\
& \tilde{f}_{\alpha}\left(i=N_{x}, j, k\right)=\tilde{f}_{\alpha}\left(i=N_{x}-1, j, k\right) .
\end{aligned}
$$

At $k=1$ and $k=N_{z}$, the same is imposed,

$$
\begin{aligned}
& \tilde{f}_{\alpha}(i, j, k=1)=\tilde{f}_{\alpha}(i, j, k=2), \\
& \tilde{f}_{\alpha}\left(i, j, k=N_{z}\right)=\tilde{f}_{\alpha}\left(i, j, k=N_{z}-1\right) .
\end{aligned}
$$

The constant pressure gradient $\nabla p$ along the $r$-direction is treated as a body force and is included in the solution procedure after the collision step and the enforcement of the above zero-derivative conditions as:

$$
\tilde{f}_{\alpha}\left(\boldsymbol{x}_{i}, t\right)=\tilde{f}_{\alpha}\left(\boldsymbol{x}_{i}, t\right)-w_{\alpha} \frac{3}{c^{2}} \frac{d p}{d x} \boldsymbol{e}_{\alpha} \cdot \hat{\boldsymbol{x}}
$$

where $\hat{\boldsymbol{x}}$ is the unit vector along the $x$-axis. On the solid walls $(y=0$ and $y=H)$, Eqs. (1.9) $-(1.11)$ are used. The exact solution for the velocity is used as the velocity initial condition. The equilibrium distribution $f_{\alpha}^{(e q)}$ function based on the exact solution for the velocity profile is used as the initial condition for $f_{\alpha}$. The pressure gradient is set to $\frac{d p}{d x}=-1.0 \times 10^{-6}$. All computations are carried out using double precision.

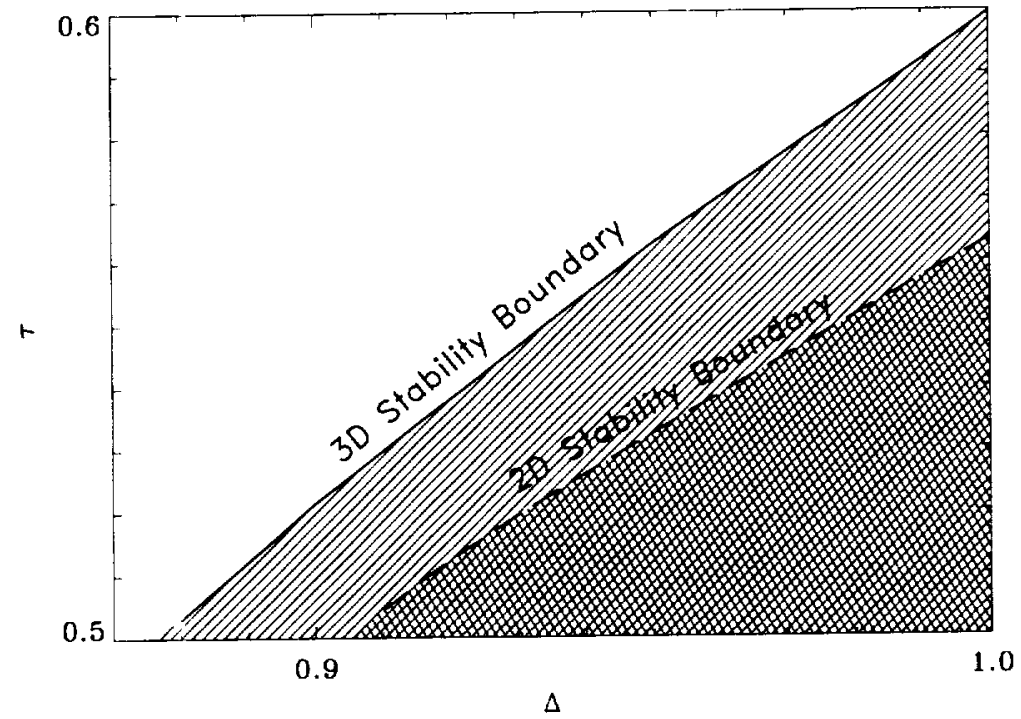

Fig. 2. Stability boundary of FH's scheme in a square duct flow for $\Delta$ near 1. Shaded areas under stability boundaries are unstable regions.

It was found that the computations are stable for $\tau$ close to 0.5 (for example, $\tau=0.505$ ) as long as $\Delta$ is not too close to unity (for example, $\Delta \leq 0.87$ ). When $\Delta$ is equal to 1 , stable computation can only be carried out for $\tau$ no smaller than 0.6. Fig. 2 shows the stability bound for the channel flow simulation with a system size $N_{x} \times N_{y} \times N_{z}=5 \times 35 \times 5$, near $\Delta=1$. Also shown by the dashed line is the stability-instability 
boundary for the channel flow simulation using D2Q9 model and with a system size $N_{x} \times N_{y}=5 \times 35$, near $\Delta=1$. It is clear that similar behavior exists in both 2-D and 3-D channel flow simulations. When the computation for the pressure driven flow in a square duct was carried out using the D3Q19 formulation, a similar stability bound was encountered.

Ideally, one would like to use a fixed value of $\tau$ for the entire range of $0 \leq \Delta \leq 1$ in a simulation. Computational stability would then require the use of $\tau$ around 0.6 , instead of a value that is close to 0.5 , which makes it difficult to simulate a lower viscosity, or higher Reynolds number flow. To overcome the restriction imposed by the numerical stability requirement due to interpolation, it would be useful if one could decrease the value of $\chi=(2 \Delta-1) / \tau$ given by Eq. (1.11a). This can be accomplished by using

$$
u_{b f}=\left(1-\frac{3}{2 \Delta}\right) u_{f}+\frac{3}{2 \Delta} \boldsymbol{u}_{w} \quad \text { and } \quad \chi=\frac{2 \Delta-1}{\tau+1 / 2} \quad \text { for } \Delta \geq 1 / 2 .
$$

That is, the velocity $\boldsymbol{u}_{b f}$ is evaluated at $\left(\boldsymbol{x}_{b}+\boldsymbol{e}_{\alpha} / 2\right)$, instead of at $\boldsymbol{x}_{b}$, using the information at $\boldsymbol{x}_{f}$ and $\boldsymbol{x}_{w}$ through linear extrapolation.

With Eq. (2.4) replacing Eq. (1.11a), the channel flow simulations using D3Q19 lattice model are carried out again for $\Delta$ from 0.85 to 1.0. Satisfactory results for the velocity profiles are obtained for $\tau=0.505$ with $N_{x} \times N_{y} \times N_{z}=5 \times 35 \times 5$ in terms of computational stability. For $\Delta<0.85$, the accuracy of the solutions using Eqs. (1.11a) and (2.4) is the same when the computations are stable.

\section{Results and Discussions.}

3.1. Fully Developed Flow in a Square Duct. For fully developed flow inside a square duct of height $H$ defined by the region $-a \leq y \leq a$, and $-a \leq z \leq a$, where $a=H / 2$, the axial velocity profile can be found in Ref. [28, p. 123]:

$$
u_{x}(y, z)=\frac{16 a^{2}}{\mu \pi^{3}}\left(-\frac{d p}{d x}\right) \sum_{k=0}^{\infty}(-1)^{k}\left[1-\frac{\cosh \left(\frac{(2 k+1) \pi z}{2 a}\right)}{\cosh \left(\frac{(2 k+1) \pi}{2}\right)}\right] \frac{\cosh \left(\frac{(2 k+1) \pi y}{2 a}\right)}{(2 k+1)^{3}}
$$

Figure 3 compares the exact axial velocity profiles at $z=0$ and the LBE-based solution with $\Delta=0.2$ and $H=2 a=32.4$. A total of $N_{x} \times N_{y} \times N_{z}=13 \times 35 \times 35$ grid points are used. The pressure gradient is $\frac{d p}{d x}=-1.0 \times 10^{-6}$ and $\tau=0.52$. The nineteen-velocity model is used in the simulations. Excellent agreement was obtained.

Figure 4a shows the dependence of relative $L_{2}$-norm error,

$$
E_{2}=\sqrt{\frac{\int_{0}^{H} \int_{0}^{H}\left[u_{\mathrm{LBE}}(y, z)-u_{\text {exact }}(y, z)\right]^{2} d y d z}{\int_{0}^{H} \int_{0}^{H}\left[u_{\text {exact }}(y, z)\right]^{2} d y d z}},
$$

on the duct height or the lattice resolution $H=N_{y}-3+2 \Delta$. The integral is evaluated by the trapezoidal rule. As was demonstrated by Mei et al. [20], the boundary treatment results in second order convergence for 2-D channel flow. Fig. 4a clearly shows that the total error (from both the flow field and the boundary condition) of the LBE solution in 3-D flow decays quadratically.

Figure $4 \mathrm{~b}$ shows the relative $L_{2}$-norm error $E_{2}$ as a function of $\Delta$ in the duct flow using $13 \times 35 \times 35$ grid points and $\tau=0.52$. For the purpose of comparison, the relative $L_{2}$-norm error in the 2-D channel flow simulation using the D2Q9 model with $N_{y}=35$ and $\tau=0.52$ is also shown. The relative error is larger in 


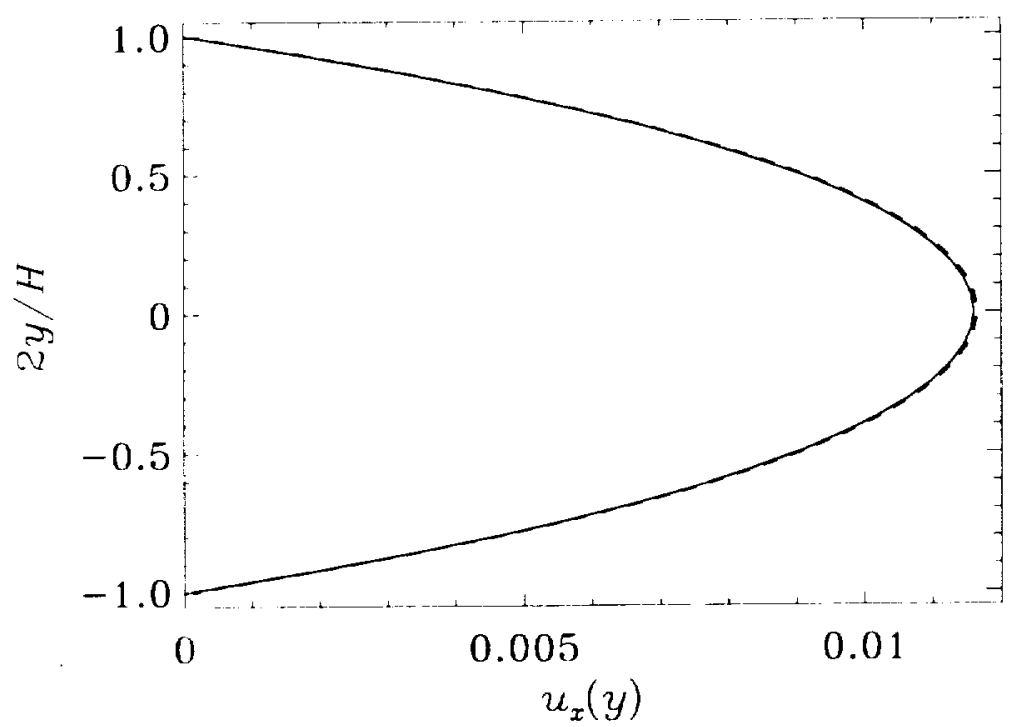

Fig. 3. Comparison of axial velocity profiles in a pressure-driven square duck flow at $z=0$ between the exact solution (solid line) and the $L B E$ solution (dashed line) with $\Delta=0.2, d p / d x=-1.0 \cdot 10^{-6}, \tau=0.52$, and $H=32.4$.

3-D duct flow than in the 2-D channel flow. Nevertheless, the error exhibits the same qualitative behavior in both 2-D and 3-D as a function of $\Delta$.

It should be noted that the accuracy of the D2Q9 model and the D3Q19 model is different in the sense that beyond the conserved moments (density and momentum in athermal fluids), these two models have different accuracy in preserving higher order moments (fluxes) [13, 14]. The D2Q9 model preserves all the moments up to the second order in momentum space, which include momentum fluxes, and maintains the isotropy of these moments, whereas the D3Q19 model can preserve density and momentum, but cannot maintain the same accuracy and isotropy of the fluxes as the D2Q9 model. The only 3-D equivalent of the D2Q9 model in terms of accuracy of the moments is the D3Q27 model $[13,14]$.

3.2. Simulation Results for 3-D Lid-Driven Cavity Flows. Lid-driven cavity flow has been studied extensively in the CFD community. Most research has been focused on 2-D problems. Limited numbers of reliable numerical results for steady state 3-D cavity flows have been obtained in the past several years. In this study, the multi-block finite difference solution of the NS equations obtained recent by Salom [26] is used to compare with the present LBE based results.

The size of the cavity is $H^{3}$, the number of grid is $N_{x} \times N_{y} \times N_{z}$, and $N_{x}=N_{y}=N_{z}$. The driving lid is placed at $y=H$, moving along the direction of $x$-axis with a speed $U=0.1$ in lattice units. Figure $5 \mathrm{a}$ compares profiles of horizontal velocity $u_{x}(y)$ obtained using $33 \times 33 \times 33$ lattices with the solution to the NS equations at $x / H=z / H=0.5$ for Re $=400$. All three LBE models (D3Q15, D3Q19, and D3Q27) are used. For the fifteen-velocity model, the computation becomes unstable and blows up at this Reynolds number with $33^{3}$ lattice resolution and $\Delta=0.5$. For $\Delta=0.5$, the nineteen-velocity model and the twentyseven-velocity model give very similar $u_{x}(y)$ profiles and both under-predict slightly the magnitude of the minimum in the profiles. The 19-velocity model is also used with $\Delta=0.25$; there is a slight overshoot in the velocity profiles in comparison to the results in Ref. [26]. Fig. 5b compares $u_{x}(y)$ profiles obtained using the fifteen-velocity and 19-velocity lattice models on the $67^{3}$ lattice grids and $\Delta=0.5$ with the NS solution [26] at $x / H=z / H=0.5$ for $\operatorname{Re}=400$. Excellent agreement is observed. Clearly, the nineteen-velocity model is 

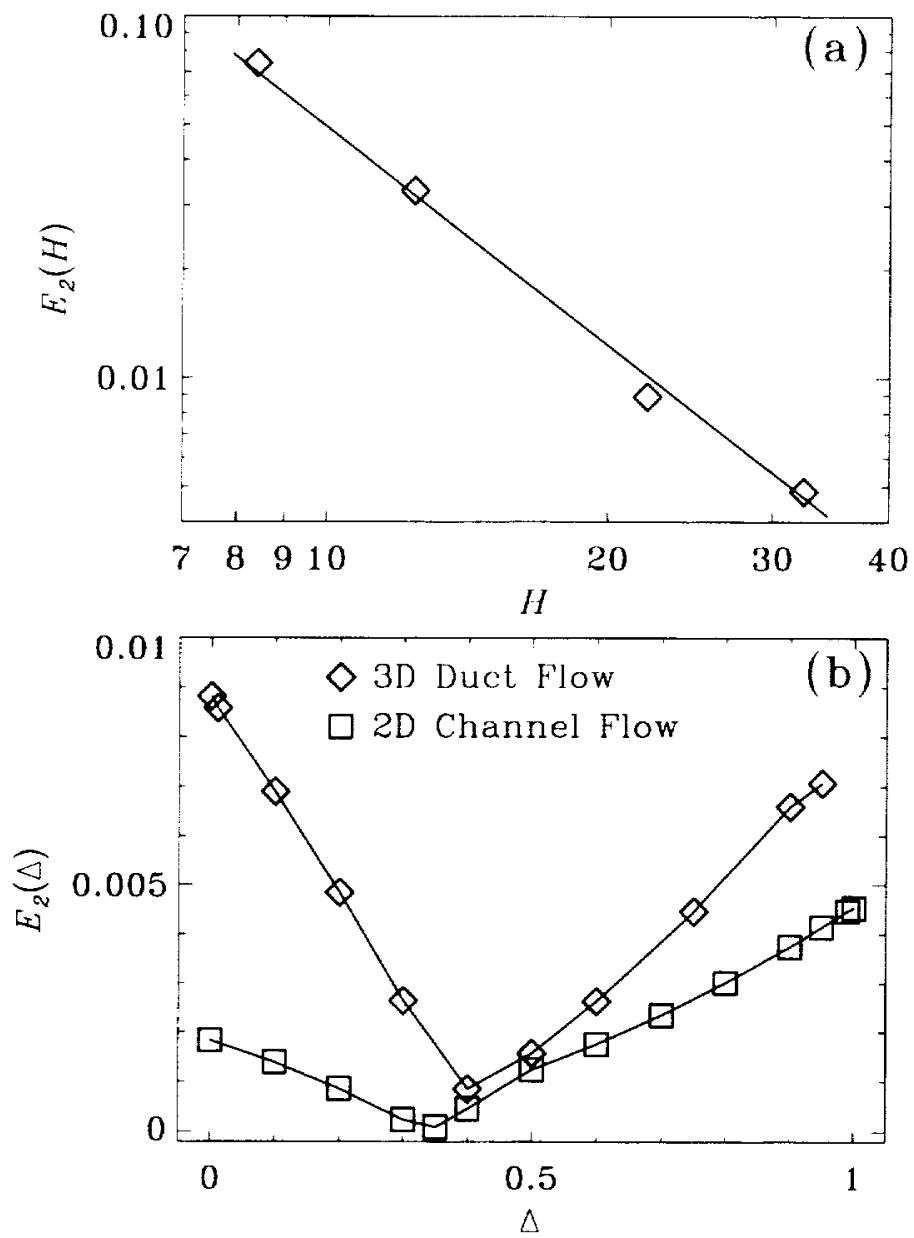

FIG. 4. Error behaviors in a pressure-driven square duck flow with $d p / d x=-1.0 \cdot 10^{-6}, \tau=0.52$. (a) Dependence of the relative $L_{2}$-norm error on the lattice resolution $H=N_{y}-3+2 \Delta$, with $\Delta=0.2$. The straight line is a least-square fit of the data (symbols) with a slope of -2 . (b) Relative $L_{2}$-norm error as a function of $\Delta$ for the 3-D duct flow (solid line) and the 2-D channel flow simulations.

superior to the fifteen-velocity model. Although the fifteen-velocity model requires $21 \%$ less CPU time and storage than the nineteen-velocity model per lattice, it is not as robust as the nineteen-velocity model and may actually require more CPU time and memory to obtain a reasonable solution since more lattice points are clearly needed.

It should be noted that stability property of the nineteen-velocity model and the fifteen-velocity model are significantly different. All LBE models have inherent spurious invariants because of their simple dynamics [18]. However, the stability of the LBE models, which is very much affected by these spurious invariants, differs from one model to another, and also depends on other factors such as boundary conditions and the local Reynolds number [18]. Among the three 3-D LBE models (D3Q15, D3Q19, and D3Q27), the D3Q15 model is the least isotropic and therefore is more prone to numerical instability. This is independently verified in a recent work by Kandhai et al. [16]. It was observed that the D3Q15 model may induce artificial checkerboard invariants which are the eigenmodes of the linearized LBGK collision operator at wave vector $k=\pi$; this can cause spatial oscillations to develop in the flow field at high Reynolds number [18]. Although it was pointed out that the presence of solid walls can suppress the oscillation in certain cases, the solid 

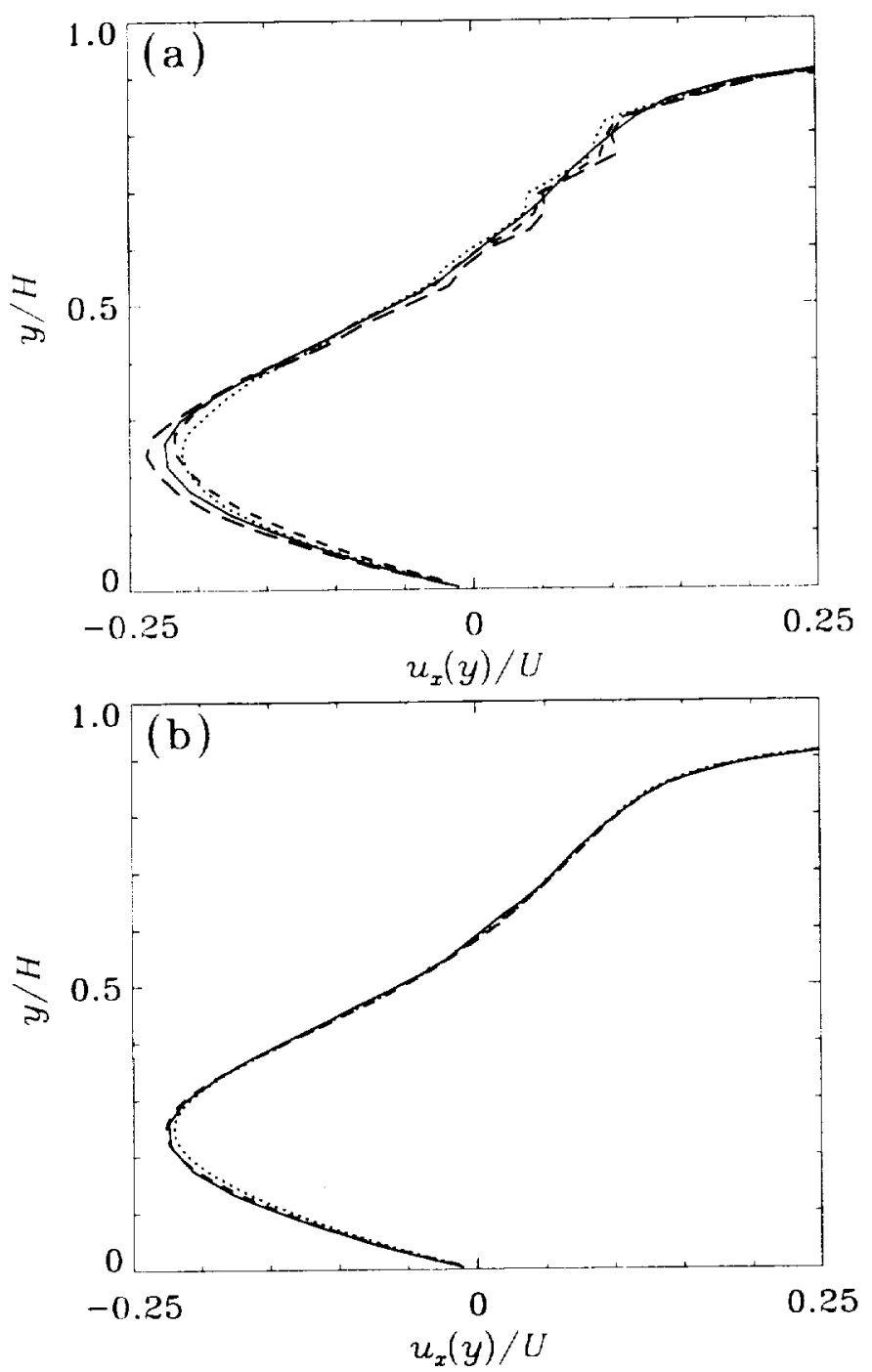

FIG. 5. 3-D lid-driven cavity flow. Normalized horizontal velocity profiles $u_{x}(x, y, z) / U$ at $x / H=z / H=0.5$, with rm $R e=400, U=0.1$. The Navier-Stokes solution (solid line) from Ref. [26] are compared with the following LBE solutions. (a) $N_{x} \times N_{y} \times N_{z}=33^{3}$, the nineteen-velocity $L B E$ solutions with $\Delta=0.5$ (dashed line) and $\Delta=0.25$ (long dashed line), and the twenty-seven-velocity $L B E$ solutions with $\Delta=0.5$ (dotted line). (b) $N_{x} \times N_{y} \times N_{z}=67^{3}$, the nineteen-velocity (dashed line) and fifteen-velocity (dotted line) $L B E$ solutions with $\Delta=0.5$.

walls in the present case actually excite the oscillation by producing a shear stress singularity at the two corners between the moving and stationary walls. Clearly, the D3Q19 model is better suited to handle flow singularities than the D3Q15 model in this case.

Figure 6a compares the profiles of transversal velocity $u_{y}(x)$ obtained from various 3-D LBE models using $33^{3}$ lattices (grids) with the NS solution at $y / H=z / H=0.5$ for $\operatorname{Re}=400$. For $\Delta=0.5$ we found that the results from the twenty-seven-velocity model deviate more from the NS results of Ref. [26] than the results of the nineteen-velocity model with the same resolution in the spatial region $0.1<x / H<0.6$. Both models under-predict the extrema of the velocity profile compared to the NS solution of Ref. [26]. For $\Delta=0.25$, the results of the nineteen-velocity model slightly over-predict the extrema, also shown in Fig. $6 \mathrm{a}$. However the difference is relatively smaller in both cases. Figure $6 \mathrm{~b}$ shows similar results of velocity profile 
with a resolution of $67^{3}$ grid points and the same Reynolds number $\mathrm{Re}=400$. With $67^{3}$ lattice resolution, the result of the fifteen-velocity model significantly differs from the result of the nineteen-velocity model and that of the NS solution in Ref. [26]. These comparisons further suggest that the nineteen-velocity model is better than the fiften-velocity model in terms of accuracy and stability, and better than the twenty-sevenvelocity in terms of computational efficiency. The nineteen-velocity model represents a good compromise in terms of both computational efficiency and reliability.
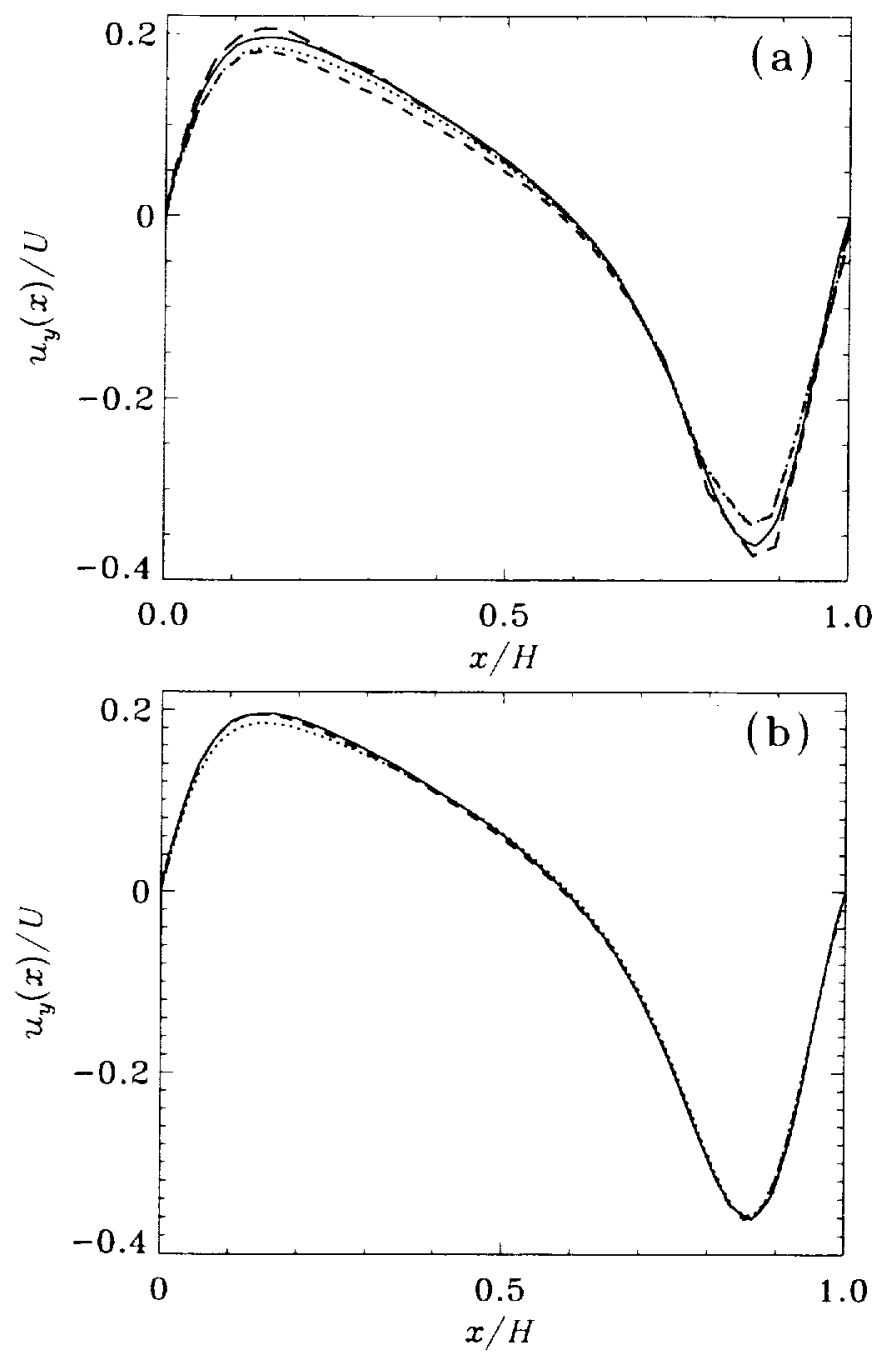

FIG. 6. 3- $D$ lid-driven cavity flow. Normalized transversal velocity profiles $u_{y}(x, y, z) / U$ at $y / H=z / H=0.5$, with $r m R e=400, U=0.1$. The Navier-Stokes solution (solid line) from Ref. [26] are compared with the following LBE solutions. (a) $N_{x} \times N_{y} \times N_{z}=33^{3}$, the nineteen-velocity LBE solutions with $\Delta=0.25$ (long dashed line) and $\Delta=0.5$ (dotted line), and the twenty-seven-velocity LBE solution with $\Delta=0.5$ (dashed line). (b) $N_{x} \times N_{y} \times N_{z}=67^{3}$, the nineteen-velocity (dashed line) and fifteen-velocity (dotted line) $L B E$ solutions with $\Delta=0.5$.

Figures 7 and 8 show the effect of Reynolds number (from 100 to 2000) on the profiles of horizontal velocity $u_{x}(y)$ and transversal velocity $u_{y}(x)$ at $x / H=z / H=0.5$ based on the D3Q19 model. For $\operatorname{Re}=100$, 400 and $1000, \Delta=0.5$ is used. It is worth noting that for $\operatorname{Re}=2000$, the system size of $67^{3}, U=0.1$, and $\tau=0.50325$, the LBE simulation with $\Delta=0.5$ eventually becomes unstable, although the steady state result of $\operatorname{Re}=1000$ is used as the initial condition for $\operatorname{Re}=2000$. When $\Delta=0.25$ is used on the $67^{3}$ lattice system, 
no computational instability occurs and the steady state solution is obtained. Weak spatial oscillation in the $u_{x}(y)$ and $u_{y}(x)$ velocity profiles was observed for $\mathrm{Re}=2000$, which indicates that further increase in Re would require better spatial resolution. It is also worth pointing out that when FH's boundary condition [8] is used for $\operatorname{Re}=2000$ with $\Delta=11.25$, the solution eventually blows up even when converged results (based on the present boundary condition for $\Delta=0.25)$ at $\mathrm{Re}=2000$ are used as the initial condition.

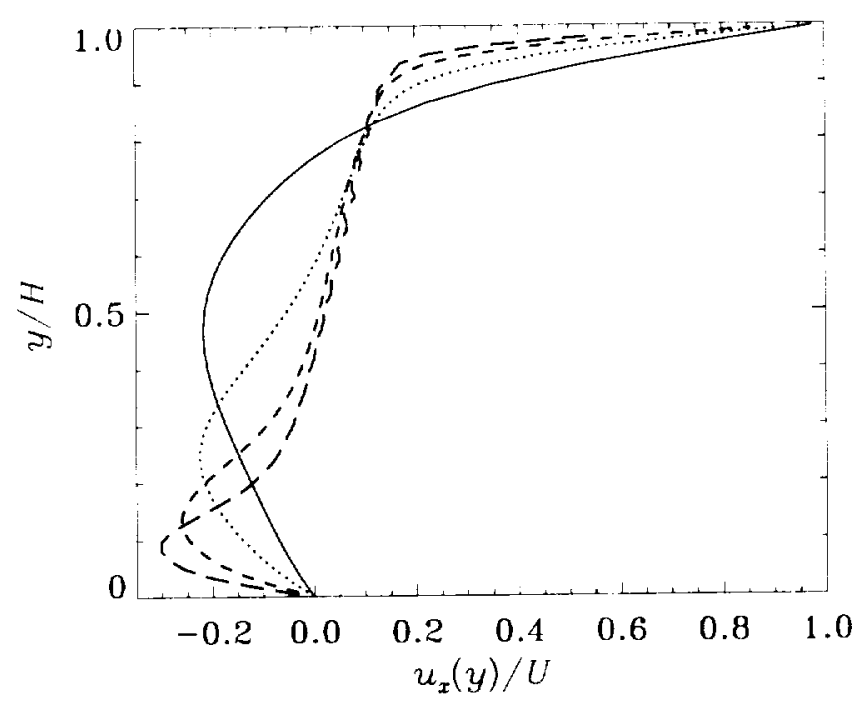

FIG. 7. 3-D lid-driven cavity flow. Effect of Reynolds number Re on the normalized horizontal velocity profiles $u_{x}(x, y, z) / U$ at $x / H=z / H=0 . \pi$. The results are obtain by using 1$) 3 Q 19$ LBE model with $U=0.1$, Re $=100$, $N_{x} \times N_{y} \times N_{z}=33^{3}, \Delta=0.5$ (solid line); $U=0.1$, Re $=400, N_{x} \times N_{y} \times N_{z}=67^{3}, \Delta=0.5$ (dotted line); $U=0.1$, $\operatorname{Re}=1000, N_{x} \times N_{y} \times N_{z}=67^{3}, \Delta=0.5$ (dashed line); and $U=0.1, \operatorname{Re}=2000, N_{x} \times N_{y} \times N_{z}=67^{3}, \Delta=0.25$ (longdashed line).

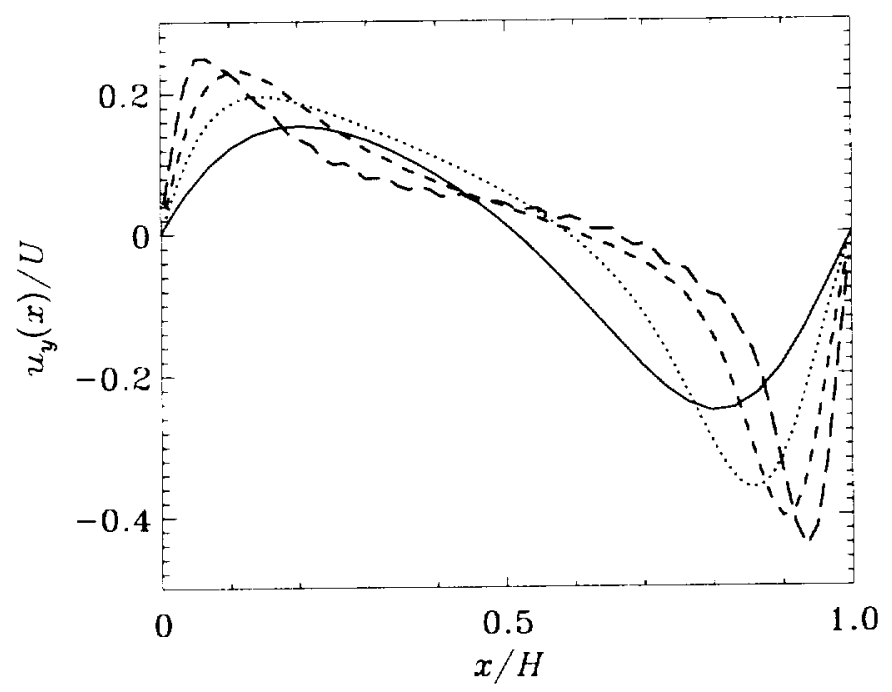

FIG. 8. 3-D lid-driven cavity flow. Effect of Reynolds number Re on the normalized transversal velocity profiles $u_{y}(x, y, z) / U$ at $y / H=z / H=0.5$. The results are obtain by using D3Q19 LBE model with $U=0.1$, Re $=100$, $N_{x} \times N_{y} \times N_{z}=33^{3}, \Delta=0.5$ (solid line) $U=0.1, \operatorname{Re}=400, N_{x} \times N_{y} \times N_{z}=67^{3}, \Delta=0.5$ (dotted line); $U=0.1$, $\operatorname{Re}=1000, N_{x} \times N_{y} \times N_{z}=67^{3}, \Delta=0.5$ (dashed line); and $U=0.1, \operatorname{Re}=2000, N_{x} \times N_{y} \times N_{z}=67^{3}, \Delta=0.5$ (long dashed line). 
3.3. Fully Developed Flows inside a Circular Pipe. Figure 9 shows the discretized domain and the boundary nodes $x_{b}$ (denoted by solid symbols) for flow inside a circular pipe of radius $R=9.5$ lattice units. Geometrically, the LBE simulation of the pipe flow differs from that of the duct flow in that the fraction of the intersected link $\Delta$ is not constant over the entire boundary. As seen in Fig. 4b, computational error can vary with $\Delta$ in the duct flow and the difference in the error can easily be as large as a factor of four for $0 \leq \Delta \leq 1$. Furthermore, the error is the smallest when $\Delta$ is between 0.3 to 0.6 . Hence, it is reasonable to expect that the overall error in the solution will depend on the distribution of $\Delta$ in the entire set of $\Delta$.

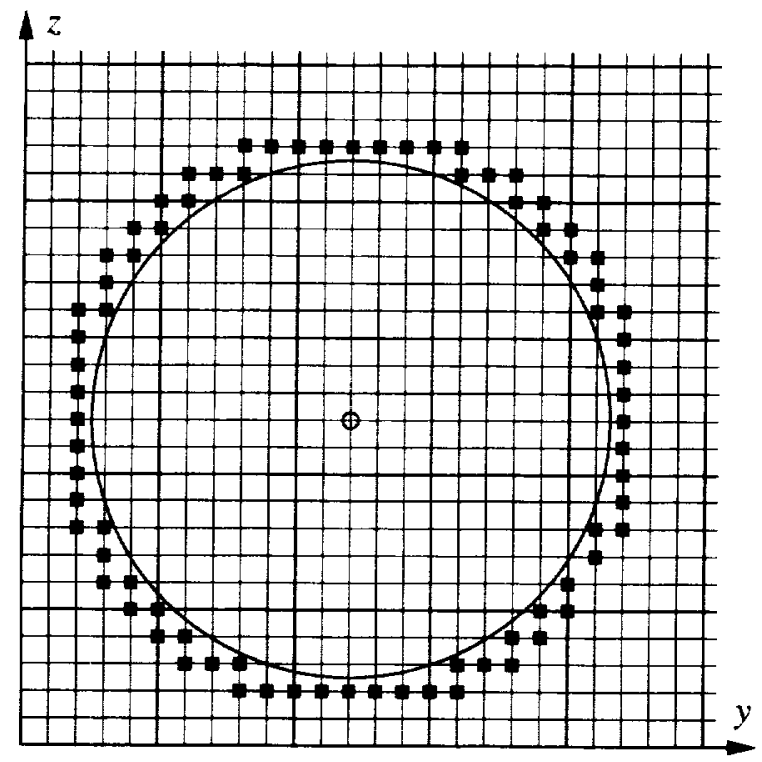

FIG. 9. Schematic for the boundary nodes $x_{b}$ (solid symbols) in yz plane in the pressure-driven in a pipe of radius $R=9.5$.

Figure 10 shows the relative $L_{2}$-norm error for the axial velocity profile defined as

$$
E_{2}=\sqrt{\frac{\sum_{\left(y_{j}, z_{k}\right) \in \Omega}\left[u_{\mathrm{LBE}}\left(y_{j}, z_{k}\right)-u_{\text {exact }}\left(y_{j}, z_{k}\right)\right]^{2}}{\sum_{\left(y_{j}, z_{k}\right) \in \Omega}\left[u_{\text {exact }}\left(y_{j}, z_{k}\right)\right]^{2}}},
$$

where $\Omega$ is the set of the discrete lattice grids inside the pipe, as a function of radius $R$ for $R=3.5,4.5$, $5.5,9.5,13.5,18.5$ and 23.5. The pressure gradient is $\frac{d p}{d x}=-1.0 \times 10^{-6}$ and $\tau=0.52$. It is noted that each simple summation in Eq. (3.3) is slightly less than the exact integration over the entire circle due to the discretization. To ensure that such a treatment does not affect the qualitative behavior of the error measurement, the centerline axial velocity, $u_{c}$, is also compared with the exact solution and the error is defined as:

$$
E_{c}=\frac{\left|u_{c, \text { LBE }}-u_{c, \text { exact }}\right|}{\left|u_{c, \text { exact }}\right|}
$$

It is seen that $E_{c}$ behaves very similarly to $E_{2}$ and both are non-monotonic. This oscillatory behavior could be due to the difference in the distribution of $\Delta$, which in turn results in the difference of the dissipation due to the interpolation around the boundary. Shown also in Fig. 10 is the error $E_{2}$ of the square duct flow solution (with $\Delta=0.2$ ) as a function of equivalent radius $H / \pi^{1 / 2}$, which exhibits a quadratic convergence. Despite the non-monotonic behavior, it can still be seen that on average, $E_{2}$ and $E_{c}$ decay quadratically 
with increasing radius and the accuracy in the pipe flow simulation is comparable to that in the square duct How simulation.

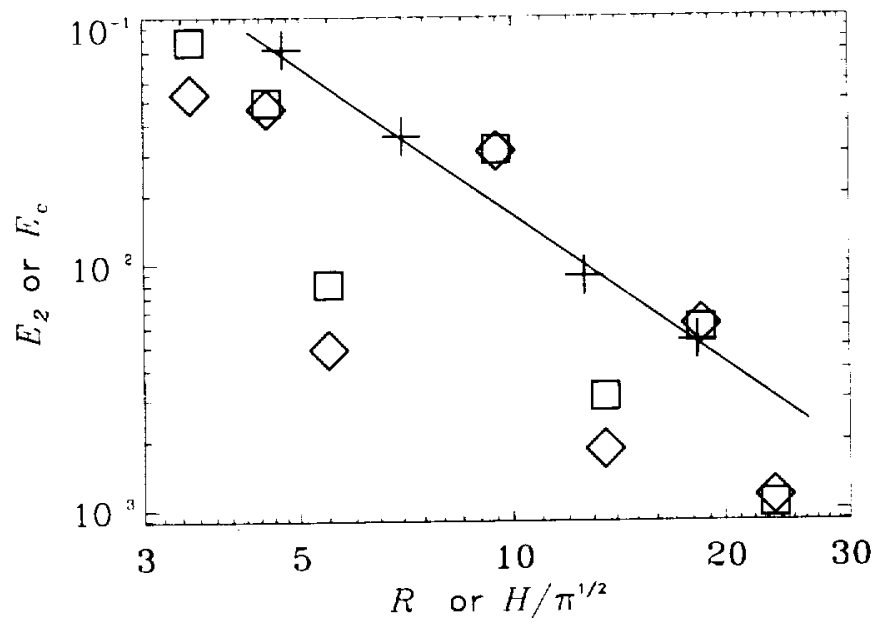

FIG. 10. Variation of relative errors $E_{2}$ and $E_{c}$ in velocity profiles as a function of the pipe radius $R$ or the equivalent radius $H / \pi^{1 / 2}$ for a square duct. Shoun are the $E_{2}(R)(\square)$ and $E_{c}(R)(\diamond)$ for the pressure-driven pipe fiow, and $E_{2}\left(H / \pi^{1 / 2}\right)$ (+) for the pressure-driven square-duct flow. The straight line is a least-square fit of $E_{2}\left(H / \pi^{1 / 2}\right)$ with a slope of -2 .

Figure 11 shows the axial velocity profiles in the pipe for $R=3.5,5.5,9.5$, and 13.5 in comparison with the exact solution. Even for a very small radius $R=3.5$, the LBE solution agrees with the exact solution remarkably well. A noticeable discrepancy in the velocity profile at $R=9.5$ is also observed in $E_{2}$ and $E_{c}$ shown in Fig. 10.

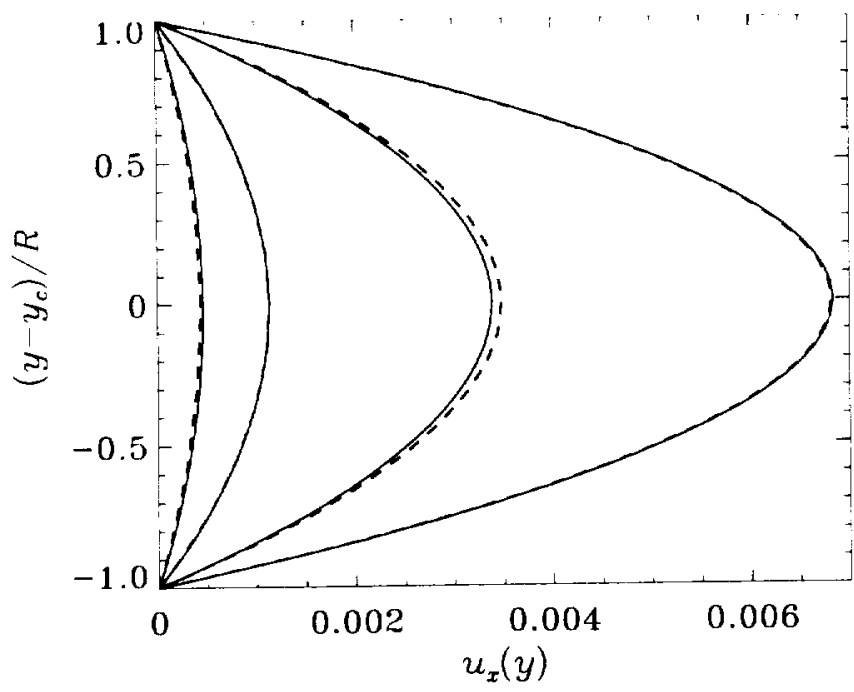

FIG. 11. Comparison of the axial velocity profiles between the $L B E$ solutions (dashed lines) and the analytic solutions (solid lines) for the pressure-driven pipe flow. Shown in the figure (from left to right) are solutions with $R=3.5,5.5,9.5$, and 13.5. $d p / d x=-1.0 \cdot 10^{-6}, \tau=0.52$

3.4. Simulation Results for a Uniform Flow over a Sphere. The conventional LBE scheme uses uniform meshes. Without local mesh refinement, it is difficult to compute the external flow over a blunt body efficiently since a large number of grid points in the far field will be wasted. As a first attempt, the 
flow over a sphere is computed within a finite region in the transversal directions.

As shown in Fig. 12, the outer boundary is placed at $y= \pm H / 2$ and $z= \pm H / 2$. At $y=-H / 2$, the lattice is $j=2$. The boundary conditions at $j=1$ for $f_{\Omega}$ 's are given by the following linear extrapolation:

$$
f_{\alpha}(i, 1, k)=2 f_{\alpha}(i, 2, k)-f_{\alpha}(i, 3, k) .
$$

The velocity at $j=2$ is set as

$$
\boldsymbol{u}(i, 2, k)=\boldsymbol{u}(i, 3, k)
$$

Similar treatment is applied at $y=+H / 2$ and $z= \pm H / 2$. The extrapolation condition given by Eqs. (3.5) and (3.6) allow the flow to leave the outer boundary. This helps to reduce the effect of the outer boundary on the flow field and on the drag force. At the inlet, a uniform velocity profile is imposed at $i=1.5$ (half way between the first and second lattice points) and Eq. (1.9) is applied to obtain the condition for $f_{\alpha}(1, j, k)$ with $\chi=0$. At the exit, a simple extrapolation is used,

$$
f_{\alpha}\left(N_{x}, j, k\right)=2 f_{\alpha}\left(N_{x}-1, j, k\right)-f_{\alpha}\left(N_{x}-2, j, k\right) .
$$

On the surface of the sphere, Eqs. (1.9), (1.10), (1.11b), and (2.4) proposed in this work are used to update the boundary conditions for $f_{\alpha}$ 's. Only the LBE nineteen-velocity model is used to simulate the flow over a sphere.
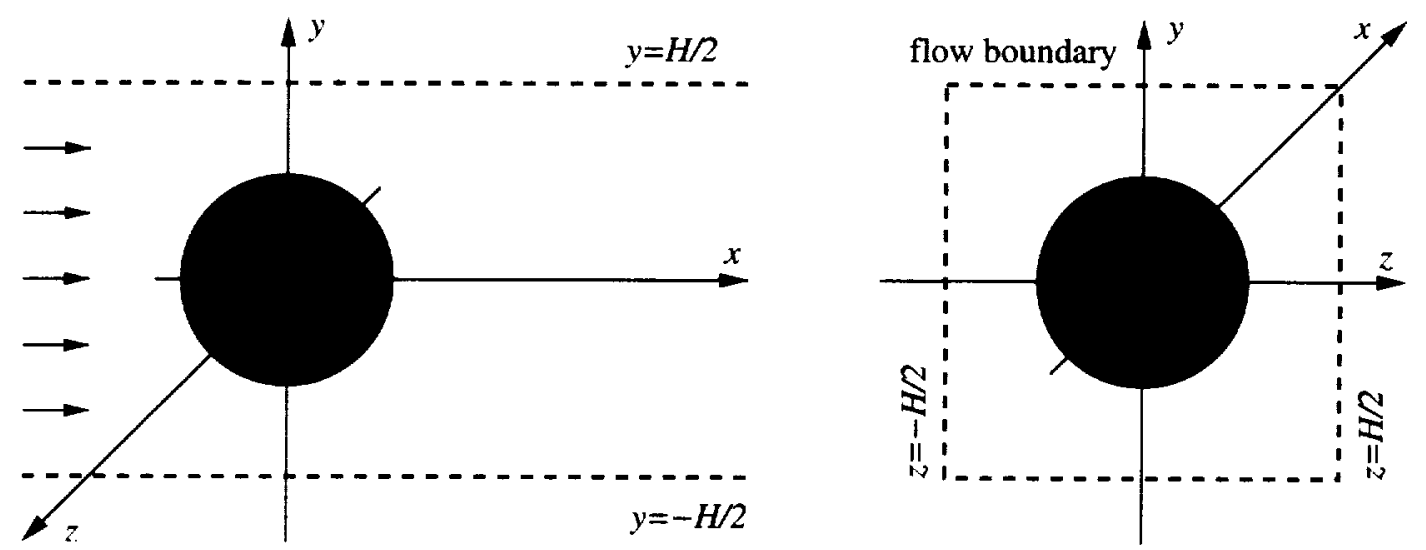

FIG. 12. Schematic for computational domain in the uniform flow past a sphere.

Figure 13 shows the velocity profile $u_{x}(y)$ based on a series of computations carried out for several values of the radius $R=3.0,3.2,3.4,3.6,3.8$ and 4.0 for $H / R=10$ at $R e=10$. The results are obtained with $\tau=0.7$. Fig. 14 compares the axial velocity profile (at $y=z=0$ ) for the same set of parameters. It is worth noting that the present LBE computation does not have sufficient resolution for the given Reynolds number. Yet the velocity profiles agree with each other accurately. The fact that we have obtained a spatially accurate solution over a range of radii strongly suggests that the present boundary condition treatment for curved geometry in the LBE method is capable of handling more complex geometries while maintaining good accuracy.

4. Concluding Remarks. Three 3-D LBE models, including the fifteen-velocity, the nineteen-velocity, and the twenty-seven-velocity model, have been assessed in terms of efficiency, accuracy, and robustness in lid-driven cavity flow. While accurate 3 -D results can be obtained by using various LBE models, the nineteenvelocity model is found to be the best for the cases investigated. The fifteen-velocity model exhibits velocity 


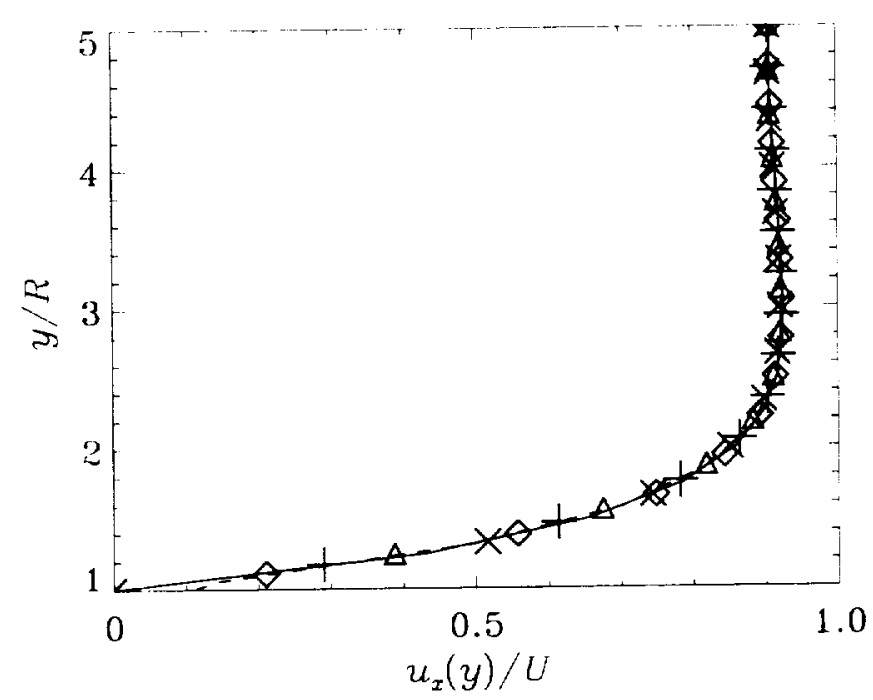

FIG. 13. Uniform flow over a sphere at $R e=10, \tau=0.7$, and $H / R=10$. Comparison of the normalized velacity profiles $u_{x}(y) / U$ at $x=z=0$ for various values for the sphere radius $R=3.0(x), 3.2(\Delta), 3.4(+), 3.6(\diamond), 3.8($ dashed line $)$, and 4.0 (solid line).

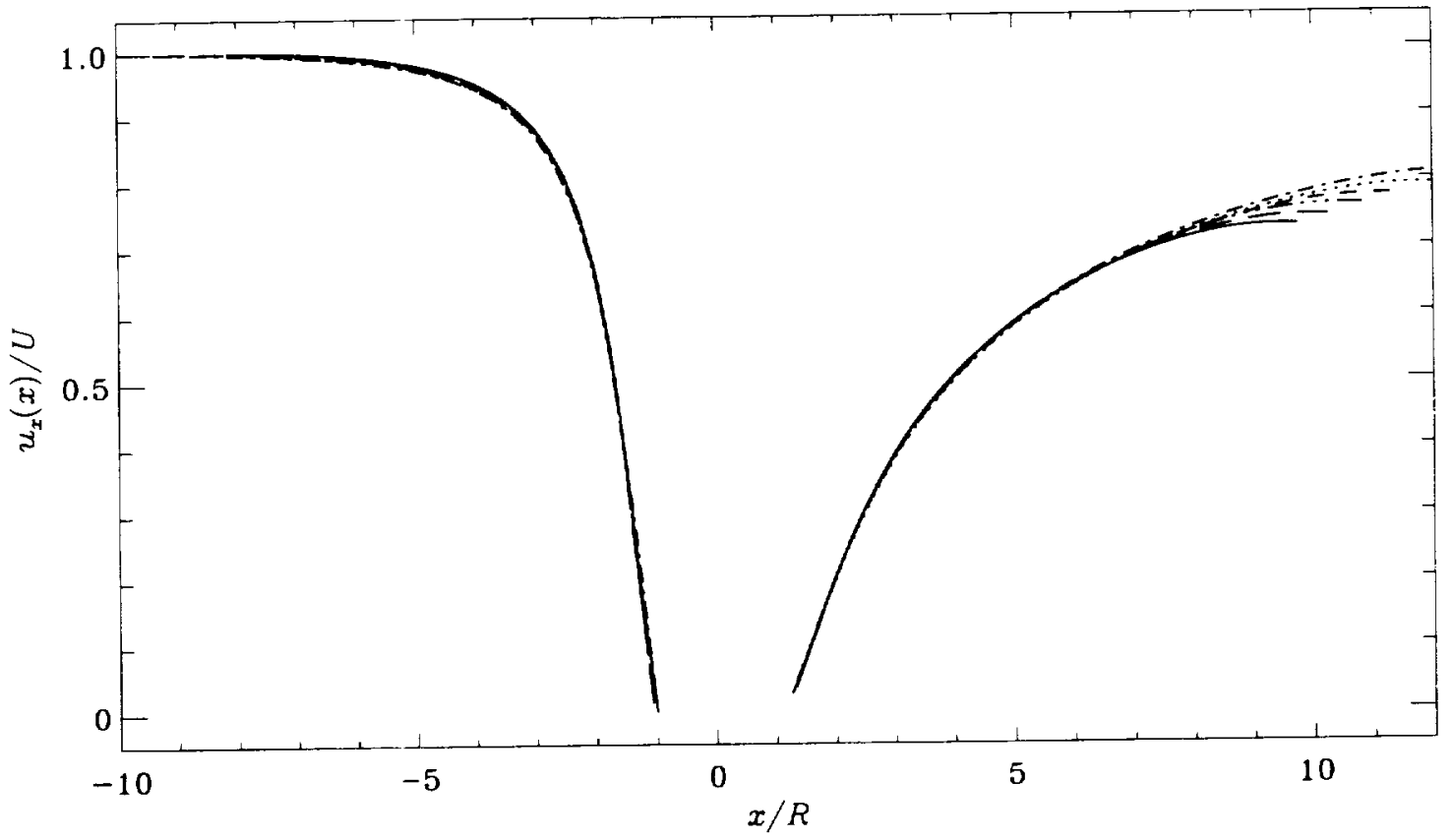

FIG. 14. Uniform flow over a sphere at $R e=10, \tau=0.7$, and $H / R=10$. Comparison of the normalized centerline at velocity profiles $u_{x}(x) / U$ (at $y=z=1$ ) for various values for the sphere radius $R=3.0$ (dash-dot line), 3.2 (dotted line), 3.4 (dashed line), 3.6 (dash dot dot dot line), 3.8 (long dashed line), and 4.0 (solid line).

oscillations and is prone to computational instability. The more complicated twenty-seven-velocity model does not necessarily give more accurate results than the nineteen-velocity model with the same spatial resolution. In this study, we have also modified the boundary condition treatment for LBE method proposed by Filippova \& Hänel [8] and Mei et al. [20] when the fraction of the intersected link on the boundary $\Delta$ is greater than one half. This improves the computational stability when $\Delta$ is close to 1 and $\tau$ close to $1 / 2$.

The simulations for flows in a square duct and in a circular pipe indicate that the current boundary 
condition treatment for curved geometries results in second-order accuracy in 3-D flows. The velocity profiles for flow over a sphere show good self-consistency of the solution over a range of sphere radii used.

Acknowledgments. This work is partially supported by NASA Langley Research Center under the program of Innovative Algorithms for Aerospace Engineering Analysis and Optimization. R. Mei also acknowledges partial support of the Engineering Research Center (ERC) for Particle Science \& Technology at the Lniversity of Florida, the National Science Foundation (EEC-9402989), and Industrial partners of the ERC. W. Shyy acknowledges partial support of AFOSR and Eglin AFB. We would like to thank Dr. O. Filippova and Prof. D. Hänel for helpful discussions, and are grateful to Dr. R. Rubinstein for his careful reading of the manuscript.

\section{Appendix A. 3-D LBE Models.}

The D3Q15 model has the following set of discrete velocities:

$$
\boldsymbol{e}_{\alpha}=\left\{\begin{array}{lll}
(0,0,0), & \alpha=0, & \text { rest particle } \\
( \pm 1,0,0) c,(0, \pm 1,0) c,(0,0, \pm 1) c, & \alpha=1,2, \ldots, 6, & \text { group I } \\
( \pm 1, \pm 1, \pm 1) c, & \alpha=7,8, \ldots, 14, & \text { group III }
\end{array}\right.
$$

and the weighting factor $w_{\alpha}$ is [24]:

$$
w_{\alpha}=\left\{\begin{array}{lll}
2 / 9, & \alpha=0, & \text { rest particle } \\
1 / 9, & \alpha=1,2, \ldots, 6, & \text { group I } \\
1 / 72, & \alpha=7,8, \ldots, 14, & \text { group III. }
\end{array}\right.
$$

The D3Q19 model has the following set of discrete velocities:

$$
\boldsymbol{e}_{\alpha}=\left\{\begin{array}{lll}
(0,0,0), & \alpha=0, & \text { rest particle } \\
( \pm 1,0,0) c,(0, \pm 1,0) c,(0,0, \pm 1) c, & \alpha=1,2, \ldots, 6, & \text { group I } \\
( \pm 1, \pm 1,0) c,(0, \pm 1, \pm 1) c,( \pm 1,0, \pm 1) c, & \alpha=7,8, \ldots, 18, & \text { group II }
\end{array}\right.
$$

and the weighting factor $w_{\alpha}$ is [14]:

$$
w_{\alpha}=\left\{\begin{array}{lll}
1 / 3, & \alpha=0, & \text { rest particle } \\
1 / 18, & \alpha=1,2, \ldots, 6, & \text { group I } \\
1 / 36, & \alpha=7,8, \ldots, 18, & \text { group II. }
\end{array}\right.
$$

The D3Q27 model has the following discrete velocities:

$$
\boldsymbol{e}_{\alpha}=\left\{\begin{array}{lll}
(0,0,0), & \alpha=0, & \text { rest particle } \\
( \pm 1,0,0) c,(0, \pm 1,0) c,(0,0, \pm 1) c, & \alpha=1,2, \ldots, 6, & \text { group I } \\
( \pm 1, \pm 1,0) c,(0, \pm 1, \pm 1) c,( \pm 1,0, \pm 1) c, & \alpha=7,8, \ldots, 18, & \text { group II } \\
( \pm 1, \pm 1, \pm 1) c, & \alpha=19,20, \ldots, 26, & \text { group III }
\end{array}\right.
$$

and the weighting factor $w_{\alpha}$ is [14]:

$$
w_{\alpha}=\left\{\begin{array}{lll}
8 / 27, & \alpha=0, & \text { rest particle } \\
2 / 27, & \alpha=1,2, \ldots, 6, & \text { group I, } \\
1 / 54, & \alpha=7,8, \ldots, 18, & \text { group II } \\
1 / 216, & \alpha=19,20, \ldots, 26, & \text { group III }
\end{array}\right.
$$

In the above, $c=\delta x / \delta t, \delta x$ and $\delta t$ are the lattice constant and the time step size, respectively. The lattice structures for the D3Q15 and D3Q19 models are shown in Fig. 15. 

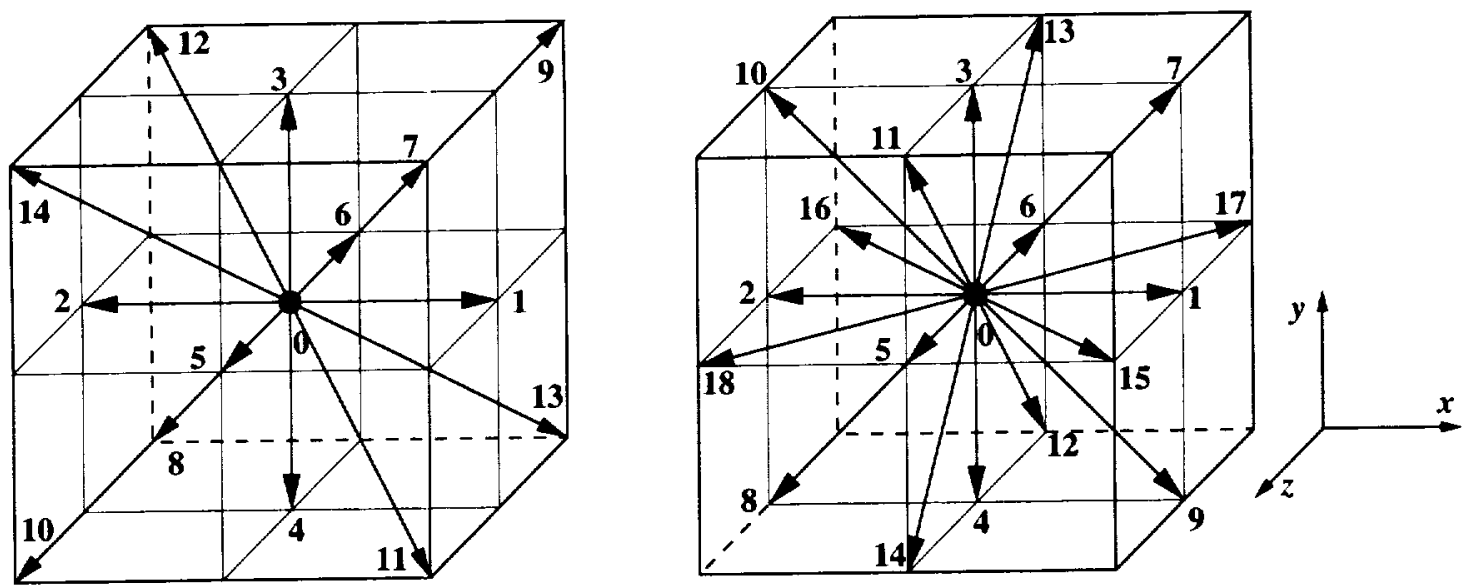

FIG. 15. Discrete velocity sets $\left\{e_{\alpha}\right\}$ for the $D 3 Q 15$ (left) and D $3 Q 19$ (right) models.

\section{REFERENCES}

[1] T. ABE, Derivation of the lattice Boltzmann method by means of the discrete ordinate method for the Boltzmann equation, J. Computat. Phys., 131 (1997), pp. 241-246.

[2] O. BeHREnd, Solid boundaries in particle suspension simulations via lattice Boltzmann method, Phys. Rev. E, 52 (1995), pp. 1164-1175.

[3] R. Benzi, S. Succi, And M. Vergassola, The lattice Boltzmann equation: Theory and applications, Phys. Rep., 222 (1992), pp. 145-197.

[4] P.L. Bhatnagar, E.P. Gross, and M. Krook, A Model for Collision Processes in Gases. I. Small Amplitude Processes in Charged and Neutral One-Component Systems, Phys. Rev., 94 (1954), pp. 511-525.

[5] H. Chen, S. Chen, And W.H. Matthaevs, Recovery of the Navier-Stokes equations using a latticegas Boltzmann method, Phys. Rev. A, 45 (1992), pp. R5339-R5342.

[6] S. Chen And G.D. Doolen, Lattice Boltzmann method for fluid flows, Annu. Rev. Fluid Mech., 30 (1998), pp. 329-364.

[7] S. Chen, D. Martínez, AND R. Mei, On boundary conditions in lattice Boltzmann method, Phys. Fluids, 8 (1996), pp. 2527-2536.

[8] O. Filippova AND D. HÄNEL, Grid refinement for lattice-BGK models, J. Computat. Phys., 147 (1998), pp. 219-228.

[9] C.A.J. Fletcher, Computational Techniques for Fluid Dynamics, Vols. I \& II (Springer-Verlag, New York, 1988).

[10] I. GinzBourg And P.M. Alder, Boundary flow condition analysis for the three-dimensional lattice Boltzmann model, J. Phys. II France, 4 (1994), pp. 191-214.

[11] I. GinzBourg AND D. D'Humières, Local second-order boundary methods for lattice Boltzmann models, J. Stat. Phys., 84 (1996), pp. 927-971.

[12] X. HE AND G. DoOLEn, Lattice Boltzmann method on curvilinear coordinates system: Flow around a circular cylinder, J. Computat. Phys., 134 (1997), pp. 306-315.

[13] X. HE AND L.-S. Luo, A priori derivation of the lattice Boltzmann equation, Phys. Rev. E, 55 (1997). pp. R6333-R6336.

[14] 
equation, Phys. Rev, E, 56 (1997), pp. 6811-6817.

[15] X. HE, Q. Zou, L.-S. Luo, AND M. Dembo, Analytic solutions and analysis on non-slip boundary condition for the lattice Boltzmann BGK model, J. Stat. Phys., 87 (1997), pp. 115-136.

[16] D. Kandhai, A. Koponen, A. Hoekstra, M. Kataja, J. Timonen, and P.M.A. Sloot, Implementation aspects of $3 D$ lattice-BGK: Boundaries, accuracy, and a new fast relaxation method, J. Computat. Phys., 150 (1999), pp. 482-501.

[17] A.J.C. LADD, Numerical simulation of particular suspensions via a discretized Boltzmann equation. Part 2. Numerical results, J. Fluid Mech., 271 (1994), pp. 311-339.

[18] P. Lallemand and L.-S. Luo, Theory of the lattice Boltzmann method: Dispersion, dissipation, isotropy, Galilean invariance, and stability, Phys. Rev. E, 61 (2000), pp. 6546-6562.

[19] L.-S. Lco, Analytic solutions of linearized lattice Boltzmann equation for simple flows, J. Stat. Phys., 88 (1997), pp. 913-926.

[20] R. MEI, L.-S. Luo, AND W. ShyY, An accurate curved boundary treatment in the lattice Boltzmann method, J. Computat. Phys., 155 (1999), pp. 307-330.

[21] R. Mei And W. ShyY, On the finite difference-based lattice Boltzmann method in curvilinear coordinates, J. Computat. Phys., 143 (1998), pp. 426-448.

[22] D.R. Noble, S. Chen, J.G. Georgiadis, and R.O. Buckius, A consistent hydrodynamic boundary condition for the lattice Boltzmann method, Phys. Fluids, 7 (1995), pp. 203-209.

[23] R. Peyret and T.D. Taylor, Computational Technique for Fluid Dynamics, Vol. II (Springer-Verlag, New York, 1983).

[24] Y.H. Qian, D. D'Humières, ANd P. LALlemand, Lattice BGK models for Navier-Stokes equation, Europhys. Lett., 17 (1992), pp. 479-484.

[25] Y.H. QIAN, S. SUCCI, AND S.A. OrsZaG, Recent advances in lattice Boltzmann computing, in Annual Reviews of Computational Physics, Vol. III, edited by D. STAUfFer (World Scientific, Singapore, 1995), pp. 195-242.

[26] J. SAlom, Numerical Simulation of Convection Phenomena Based on Domain Decomposition Techniques and Experimental Validation, Ph.D. Dissertation, Universitat Politecnica de Catalunya, Spain, 1999.

[27] W. SHYY, Computational Modeling for Fluid Flow and Interfacial Transport, corrected printing (Elsevier, Amsterdam, 1997).

[28] F.M. White, Viscous Fluid Flow (McGraw Hill, New York, 1974).

[29] D.P. Ziegler, Boundary conditions for lattice Boltzmann simulations, J. Stat. Phys., 71 (1993), pp. 1171-1177.

[30] Q. ZOU AND X. HE, On pressure and velocity boundary conditions for the lattice Boltzmann BGK model, Phys. Fluids, 9 (1997), pp. 1591-1598. 


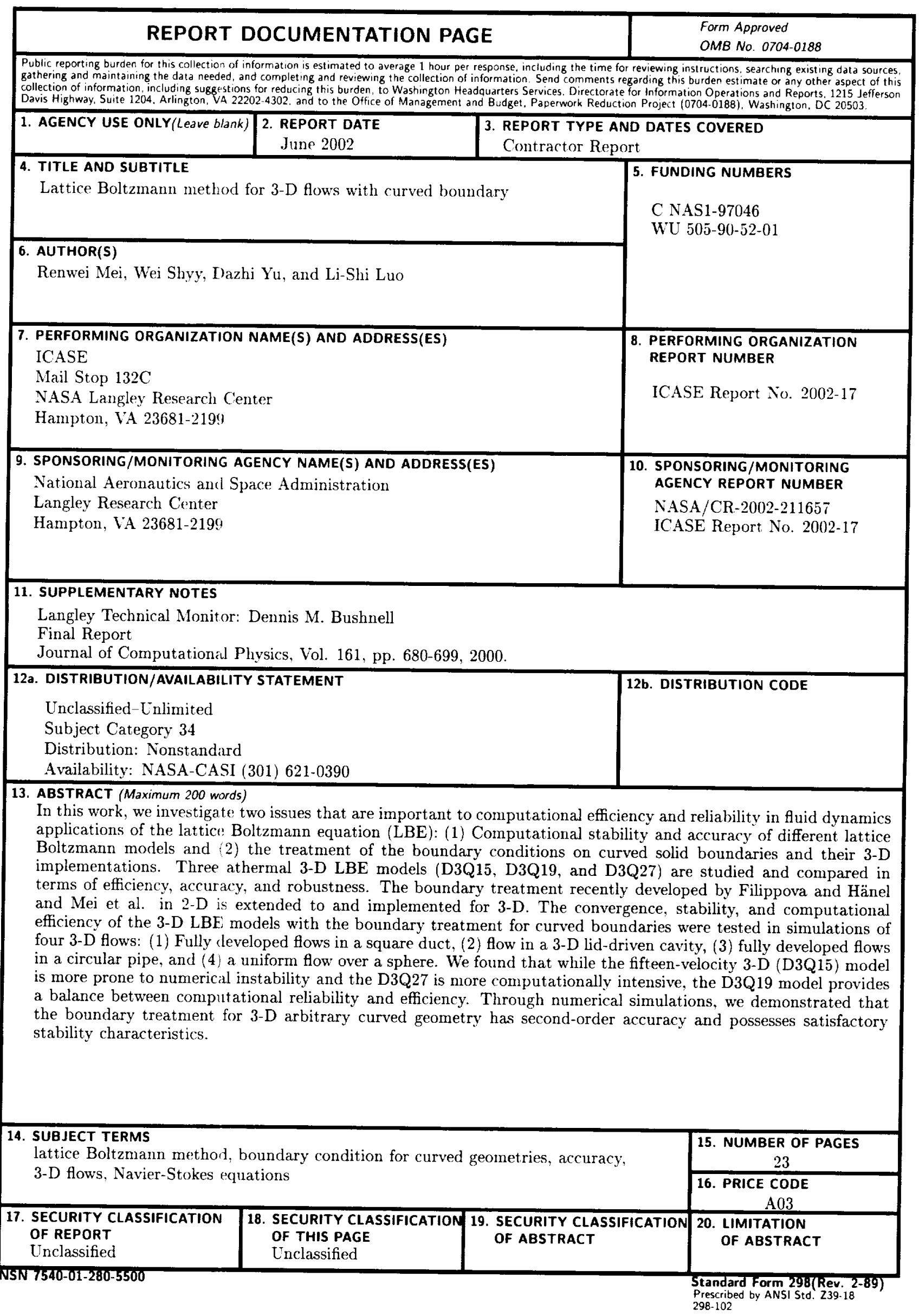


